\title{
Jerarquía de valores y práctica coral en el contexto universitario de Ecuador
}

\author{
Mónica O’Reilly Viamontes ${ }^{1}$
}

Recibido 4 de Diciembre de 2019 / Aceptado 23 Marzo de 2020

Resumen. El objetivo del presente estudio es explorar la contribución de la práctica coral en el desarrollo de los valores dentro de un grupo de cantores universitarios de Ecuador. Para tal propósito, se aplica una encuesta basada en la Teoría de los Valores de Schwartz que busca discernir cuáles valores se desarrollan según el criterio de los participantes, en mayor o menor medida, dentro del coro. De esta forma, se pudo establecer una jerarquía donde hedonismo, conformidad y benevolencia figuran como los valores que más se han desarrollado, mientras que poder y seguridad se ubican entre los valores más bajos. Así, se afianza el principio de que las prácticas musicales colectivas son idóneas para la formación de valores.

Palabras clave: música; práctica coral; valores humanos; formación integral; educación musical.

\section{[en] Hierarchy of values and choral practice in the university context of Ecuador}

\begin{abstract}
The aim of this study is to explore the contribution of choral practice in the development of values of a group of university singers from Ecuador. For this purpose, a survey based on Schwartz's Theory of Values is applied that seeks to discern which values are developed according to the criteria of the participants, to a greater or lesser extent, within the choir. In this way, it was possible to establish a hierarchy where hedonism, conformity and benevolence appear as the most developed values, while power and security are among the lowest values. Thus, the principle that collective musical practices are suitable for the formation of values is strengthened.
\end{abstract}

Keywords: music; choral practice; human values; comprehensive training; music education.

Sumario. 1. Introducción. 2. Marco teórico. 2.1. Acerca de los valores. 2.2. Acerca de la práctica coral. 3. Metodología. 4. Análisis de datos. 5. Discusión y conclusiones. 6. Referencias bibliográficas.

Cómo citar: O’Reilly, M. (2019) Jerarquía de valores y práctica coral en el contexto universitario de Ecuador. Revista Electrónica Complutense de Investigación en Educación Musical, 17, 13-20.

\section{Introducción}

Uno de los temas con mayor relevancia dentro de los cuestionamientos que plantean los foros de educación en el presente siglo se refiere a los valores y a las acciones que desde la enseñanza, se pueden realizar con relación a ellos (Parra, 2003). Es por esto que, muchas instituciones formulan estrategias para la educación en valores, estrechamente vinculada a la formación integral (Chica, 2008).

Dentro de este contexto, diversas investigaciones que examinan la relación que se establece entre los valores y el arte, demuestran que este último, no puede ser considerado únicamente como una forma de representación. El arte es también, una estrategia, un modo de actuar frente a la realidad (Cornago, 2010).

Encontramos por ejemplo que para Páez (2009), la literatura posee un papel primordial en la formación de un conjunto de valores cognitivos, éticos y estéticos que apuntan a la formación integral, mientras que, a decir de Aparicio (2009), el teatro es una fuente de conocimiento experiencial y una alternativa para la educación en valores. Sobre la capacidad del cine para reforzar conductas como un medio de desarrollo de determinados valores sociales e individuales nos habla Almenara (2003), y según Cabedo y Arriaga (2016), existen diversos estudios relacionados con la eficacia de la música para convertirse en instrumento de paz, cohesión social o aprendizaje en valores.

Universidad de Especialidades Espíritu Santo, Ecuador.

E-mail: moreillly@uees.edu.ec

Rev. electrón. complut. inves. educ. music. 17, 2020: 13-20 
Si se parte del hecho de que la música es una experiencia multidimensional con la capacidad de movilizar aspectos afectivos, físicos y mentales, de expresión, de comunicación y de conexión (Gainza, 2003), es posible comprender, como expresa Conejo (2012), que mediante ella se canalicen variadas respuestas creativas a situaciones imprevistas. En opinión del autor, permite además adiestrar la observación, la atención, la concentración, la memorización y la agilidad mental, contribuye al autoconocimiento, la reflexión, el autocontrol, el poder de decisión, la delimitación de libertades, el logro de metas y el sentido de justicia, e incluso, aminora el temor y la agresividad.

Dentro de las manifestaciones musicales, el coro es una expresión colectiva semejante a una pequeña sociedad (Lenger, 2013). Ha de considerarse que más que un grupo de cantores, es un grupo humano (Jaraba, 1989). Esta práctica demanda rigor, respeto y atención para poder lograr su cometido (Ferrer, 2009) y posee, además, según PérezAldeguer (2014a), una alta capacidad inclusiva, si se toma en cuenta la posibilidad que tienen los individuos de reformular la idea que tienen de sí mismo y de los demás, al involucrar en ella, sus emociones y sentimientos.

Es por esto que, la música es un magnífico instrumento de transmisión de valores (Conejo, 2012), y aquellos que pertenecen a un coro u orquesta, se forman en disciplina, estímulo y sensibilidad, haciendo que ellos aparezcan de forma natural, mientras aprenden a compartir (Ferrer, 2009).

Llegado a este punto vale considerar que, para comprender la contribución de los valores en la educación y su participación en los procesos educativos, es necesario entender su funcionamiento y jerarquía (Piotrowski, 2009). Si se toma en cuenta que todo ejercicio formativo, explícita o implícitamente, conlleva a una relación con los mismos. "No hay educación sin valores, por lo que la calidad y los valores de la educación dependen de los valores de la misma" (Gervilla, 2004, p.110). Por lo tanto, su estudio dentro de las prácticas educativas, conllevan al esclarecimiento de las maneras en que se manifiestan y, por ende, hacen más viables las trasformaciones dentro de los procesos de enseñanza.

Basándose en lo antes expuesto, el objetivo del presente estudio es explorar la contribución de la práctica coral en la formación de valores dentro de un grupo de cantores universitarios de Ecuador. Para ello, se aplica una encuesta que examina cuáles valores humanos se desarrollan, según el criterio de los participantes, dentro del coro, y se establece una jerarquía de los mismos basada en la Teoría de los Valores (Schwartz, 1992).

\section{Marco teórico}

\subsection{Acerca de los valores}

La complejidad de la definición de los valores ha sido abordada desde el campo de la ética, la estética, la sociología, la psicología y la pedagogía (Ojalvo, Kraftchenho, González y Rojas, 2003).

Schwartz (1992) integrando aspectos presentes en definiciones que le antecedieron, considera que los valores son creencias o conceptos que guían la conducta, que toman como referencia estados deseables que trascienden situaciones específicas y poseen un orden relativo para cada individuo o cultura (Cayón, 2008; García, Barbero y Muñoz, 2017). Los valores son estándares vinculados a las emociones y los conocimientos basados en metas abstractas, que crean un orden para la selección y evaluación de acciones, personas y eventos (Junco, Susanibar y Dutschke, 2010).

$\mathrm{Al}$ analizar las definiciones de valor, que Junco et al. (2010) sintetizan, es posible encontrar los términos concepción y creencia en la segunda mitad del siglo XX, mientras que los conceptos más cercanos a este siglo se refieren a estructuras de pensamiento o representaciones cognitivas. En general, la mayoría de los autores emplean los vocablos acción o conducta, por un lado, y por otro, deseos, preferencias e intereses.

Para Ojalvo et al. (2003), los valores se refieren a los fenómenos y objetos que poseen un sentido social positivo, que regulan la actividad humana y cuya función es tanto cognoscitiva como pragmática. Su establecimiento depende del consenso u opinión social. Según Cayón (2008), Kornblit concibe a los valores como estructuras cognitivas complejas que implican dimensiones evaluativas y conductuales, y Tunnermanh, como creencias que seleccionamos y agregamos a nuestra conducta para conducir la vida.

Por su parte, Schwartz (2012) afirma que existen diez valores básicos cuya estructura de relaciones se basa en el conflicto y congruencia. Estos valores se pueden distinguir por la motivación o meta que expresan. La búsqueda de un valor, trae aparejada el antagonismo con otro. Así se conforman dos dimensiones bipolares: Apertura al cambio/ Conservación y Autopromoción/Autotrascendencia.

Tabla. 1. Diez valores básicos (Schwartz,2012)

\begin{tabular}{|l|l|l|l|}
\hline Valor & \multicolumn{2}{|l|}{ Dimensiones opuestas } & Valor \\
\hline Autodirección & Apertura al cambio & Conservación & Seguridad \\
\cline { 1 - 1 } Estímulo & & & Conformidad \\
\cline { 1 - 1 } Hedonismo & & Tradición \\
\cline { 4 - 4 } & Autopromoción & Autotrascendencia & Benevolencia \\
\cline { 1 - 1 } Logro & & & Universalismo \\
\hline Poder & & & \\
\hline
\end{tabular}


Autodirección y estímulo pertenecen a Apertura al cambio, y seguridad, conformidad y tradición a Conservación. Poder y logro pertenecen a Autopromoción, y universalismo y benevolencia a Autotrascendencia. Hedonismo se comparte entre las dimensiones de apertura al cambio y autopromoción (Schwartz, 2012) y para su medición se incluye en Apertura al cambio (Schwartz, 2003).

Apertura al cambio expresa el pensamiento y acción independiente, la búsqueda de desafíos en oposición a Conservación, que contempla el orden, la auto restricción y la resistencia al cambio. Autopromoción incluye valores que enfatizan la búsqueda de intereses personales y estatus social, mientras que Autotrascendencia, incluye los que priorizan las necesidades colectivas y la preocupación por el bienestar de los demás (Schwartz, 2012; Expósito, 2018).

Por otro lado, en el modelo de Schwartz, los valores se disponen de forma circular, representando un ciclo motivacional. En la medida que los valores se encuentren más cercanos en cualquier dirección del círculo, mayor compatibilidad existirá entre ellos desde el punto de vista de sus motivaciones (Castro, Neto, Pena y Ribeiro, 2017; Junco et al., 2010). Por esto, aunque dentro de su teoría se delimitan 10 valores básicos, los valores adyacentes comparten énfasis motivacionales (Schwartz, 2012).

Esta teoría ha sido utilizada en investigaciones en más de 80 países tomando en cuenta que, para el autor, el perfil de valor individual promedio es una forma de caracterizar las prioridades de valor cultural y los miembros de casi todas las culturas se relacionan con estos diez valores (Schwartz, 1992).

\subsection{Acerca de la práctica coral}

La etimología de la palabra coro proviene del griego chorós y del latín chorus y hace referencia al conjunto de personas que danzaban o cantaban en un lugar cerrado. Mas allá de su diversidad de formatos, funciones y concepciones estéticas, en un coro es imprescindible el acto de cantar y el hecho de que sea en conjunto. Cantar en comunidad permanece inalterable en su concepto. Es su esencia.

Dentro de la estructura de esta práctica musical existe un elemento que, pese al transcurso del tiempo, ha perdurado: el director. En él, es importante que se combinen varias tipologías de liderazgo, e incluso, desde su rol de líder, fomente el liderazgo compartido entre los integrantes a través de la distribución de tareas (Calderón, Oriola y Gustems, 2015). En este sentido, Ordás y Martínez (2018) también observan la complejidad de relaciones que dentro de un coro se generan, cuando indican que:

Reducir la función del coreuta a un mero instrumento, hace que se pierda de vista la riqueza de las relaciones intersubjetivas que ocurren en el interior de un coro como un organismo vivo y sujeto a un intenso proceso comunicativo e interactivo. (p.8)

Aunque la figura del director es una característica que comparte el coro con otras prácticas musicales, existe un elemento que es casi restrictivo de la música vocal. Las obras escritas para voz, tanto para solista como para coro, a diferencia de las obras instrumentales, poseen un texto. El canto, involucra un texto correlacionado con la melodía (Gallardo, 2011) y su comprensión, constituye uno de los retos de la interpretación musical (Tavares, 2018).

Dentro de este proceso de construcción de la interpretación musical, donde se interrelacionan texto, ideas y música, surge la oportunidad de profundizar, no solo en cuestiones musicales, sino también en otros aspectos del ser humano. Para Hernández y Sánchez (cit. en Lorenzo, 2017), las canciones pueden emplearse para la formación de valores puesto que poseen un "discurso propio, un contenido, una letra o un mensaje" (p. 160). Adicionalmente, la interpretación de música coral, en su propósito de lograr los objetivos prefijados por y para el coro, puede constituir una forma de mejoramiento personal (Fernández, 2014).

En cuanto a los beneficios del canto coral en la etapa universitaria, Nadal, López, Fernández y Juan (2018) afirman que "crea un sentimiento profundo de igualdad y equidad entre los miembros" (p.866). Además, desarrolla competencias en los jóvenes, como la de trabajo en equipo, sujetas a ser aplicadas en otros contextos sociales y laborales (O'Reilly, 2015).

Por otro lado, Lamont, Murray, Hale y Wright-Bevans (2018) observan que el compromiso de cantar juntos en un coro influye a muchos niveles las relaciones interpersonales de los adultos mayores que lo integran. Así mismo, la labor comunitaria de una agrupación coral de adultos mayores contribuye a la percepción del valor personal y el sentido de identidad grupal (Joseph y Southcott, 2017).

\section{Metodología}

El presente estudio posee un enfoque cuantitativo de nivel descriptivo transeccional de diseño no experimental. Para ello, se tomó la totalidad de la población conformada por 31 integrantes activos y de egreso reciente, del coro de una universidad en Ecuador. En su mayoría de entre 25 y 30 años $(61,3 \%)$.

Se aplicó una encuesta basada en un cuestionario diseñado por García et al. (2017), que adapta el Schwartz Value Survey que es el primer instrumento diseñado para medir valores dentro de esta teoría (Schwartz, 2012), y uno de los más utilizados para estudiar las diferencias en los valores personales (Junco et al.,2010). 
Dentro del contexto de este estudio, los participantes fueron cuestionados acerca de cómo calificarían el grado en el que el coro ha contribuido a desarrollar un determinado número de valores, expresados mediante 50 items. Cada uno de los 10 valores básicos de Schwartz se abordaron con 5 preguntas. Para calificarlos se utilizó una escala de diferencial semántico de 7 puntos: muchísimo, mucho, bastante, algo, poco, casi nada y nada.

La validez del instrumento se obtuvo a través del juicio de 3 expertos. La confiabilidad se midió con el Alfa de Cronbach a través de un análisis factorial exploratorio AFE. El índice de adecuación muestral de Kaiser-Meyer-Olkin indica que los datos son adecuados para aplicar esta técnica y los resultados, obtenidos en el programa SPSS Versión 20, se muestran en la tabla 2. Como se puede observar, el coeficiente de Alfa de Cronbach osciló entre 0,71 y 0.9, lo cual está dentro de los parámetros aceptables para dicho indicador.

Tabla. 2. Alfa de Cronbach e índice KMO

\begin{tabular}{|l|c|c|}
\hline \multicolumn{1}{|c|}{ Variable } & Alfa de Cronbach & KMO \\
\hline Logro & 0,739 & 0,73 \\
\hline Benevolencia & 0,852 & 0,78 \\
\hline Poder & 0,716 & 0,71 \\
\hline Autodirección & 0,811 & 0,71 \\
\hline Universalismo & 0,837 & 0,76 \\
\hline Tradición & 0,873 & 0,73 \\
\hline Seguridad & 0,878 & 0,73 \\
\hline Estímulo & 0,847 & 0,70 \\
\hline Conformidad & 0,814 & 0,77 \\
\hline Hedonismo & 0,903 & 0,82 \\
\hline
\end{tabular}

Posteriormente, siguiendo los procedimientos de Schwartz, se eliminaron las diferencias individuales en el uso de la escala de las respuestas, restando la respuesta media de cada persona a sus respuestas para cada ítem. Luego, se calcularon los promedios para cada valor y cada dimensión, estableciendo así, una jerarquía relacionada con la práctica coral de la población estudiada. Para la sistematización de la información se analizaron las frecuencias porcentuales de los indicadores más significativos y se tomaron en cuenta los énfasis motivacionales que comparten los valores adyacentes.

\section{Análisis de datos}

Un primer acercamiento a los resultados mediante el análisis de los valores porcentuales más altos alcanzados en los items de forma individual, manifiesta que más de la mitad de los encuestados consideraron que el coro contribuía muchísimo a las opciones que se muestran en el siguiente gráfico (Fig.1):

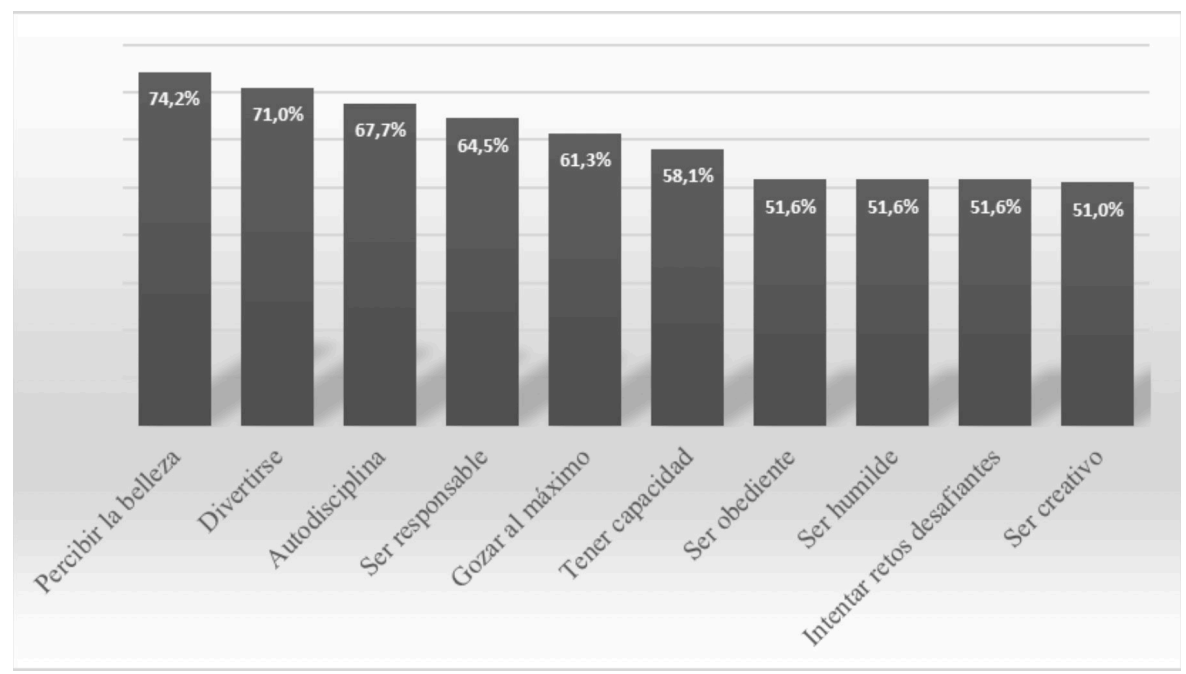

Figura 1. Porcientos mas altos alcanzados en los items 
En estos items, se representan la mayoría de los valores estudiados con al menos un indicador: universalismo (percibir la belleza), hedonismo (divertirse, gozar al máximo), conformidad (autodisciplina, ser obediente), benevolencia (ser responsable), logro (tener capacidad), tradición (ser humilde), estímulo (intentar retos desafiantes) y autodirección (ser creativo). Poder y seguridad no se encuentran entre ellos, puesto que no sobrepasaron el $38,8 \%$ de encuestados que señalaran muchísimo.

Hedonismo posee dos indicadores en los porcentajes más altos (Fig.1). Divertirse (71\%) y gozar al máximo (61,3\%). Divertirse es el único item de toda la encuesta cuyas respuestas están únicamente entre los tres puntos más altos de la escala dada. Es decir, entre bastante y muchísimo. El resto de los indicadores para este valor, fueron calificados entre algo y muchísimo. En ellos, se cuestionaba acerca de obtener placer, disfrutar la vida y tener satisfacciones.

Autodisciplina $(67,7 \%)$ y obediencia $(51,6 \%)$, que pertenecen a conformidad, también se encuentran entre los indicadores con mayor porcentaje en muchísimo, como se observa en la Figura 1. De igual manera que, ser responsable (64,5\%), indicador de benevolencia. El resto de los ítems para este valor se muestran en muchísimo de modo descendente, de la siguiente forma: ayudar a otros $(38,7 \%)$, ser leal y honesto $(32,3 \%)$ y perdonar $(16,1 \%)$.

En el caso de tradición, además de cuestionar acerca de ser humildes (51,6\%), se incluían otros indicadores relacionados con el respeto, la moderación y la devoción. Todos, con excepción de ser devoto, manifestaron cifras por encima del $60 \%$ entre mucho y muchísimo.

Una vez calculadas las medias de cada valor fue posible determinar el orden de prioridades, más allá de los porcentajes por indicadores individuales que inicialmente se tomaron en cuenta. De esta forma, es posible observar que hedonismo, ocupa el punto más alto, y seguridad, el punto más bajo (Fig.2).

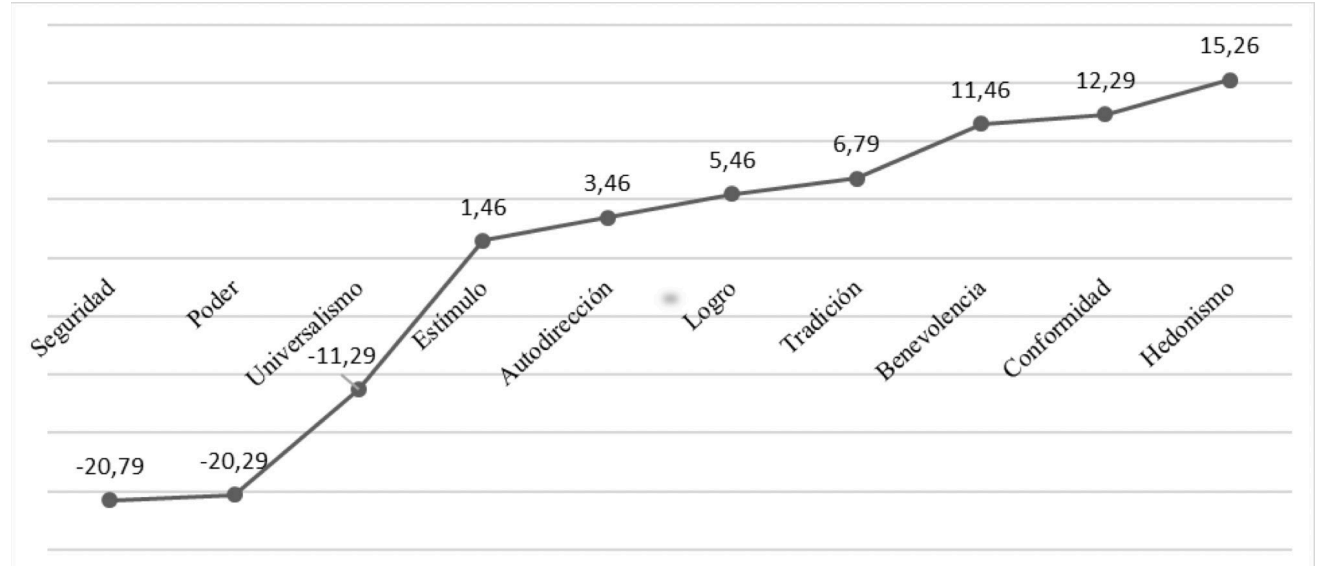

Figura 2. Jerarquía de valores para grupo de cantores

Los tres valores que se observan por debajo de hedonismo (Fig.2), conformidad, benevolencia y tradición, son valores adyacentes que comparten motivaciones. Esto denota que los encuestados consideran que el coro ha desarrollado en ellos de forma considerable, el comportamiento normativo que promueve relaciones cercanas, tomando en cuenta la relación entre conformidad y benevolencia; y un fuerte apego al grupo al que se pertenece, por la relación entre benevolencia y tradición.

A continuación, aparece logro, que contenía indicadores concernientes a la capacidad, la inteligencia, la ambición, el éxito y el hecho de ser influyente. El 58,1\% de los encuestados catalogó de muchísimo la influencia que ejercía el coro en su propósito de alcanzar capacidad. Este item, al igual que el relacionado con la ambición, no fue marcado en las tres gradaciones más bajas, por lo que puede considerarse que todos conciben esta actividad, como una forma de tener ambiciones y desarrollar sus capacidades.

Los valores que aparecen por debajo de logro, pertenecen a la misma dimensión: Apertura al cambio. Al indagar acerca de los indicadores de autodirección relacionados con las posibilidades del coro de contribuir a la independencia, libertad y curiosidad, se pudo observar que el $87 \%$ consideró que le ayudaba entre mucho y muchísimo, a elegir metas propias. De forma descendente, la contribución de esta práctica fue vista desde ser creativos $(64,5 \%)$ hasta ser independiente $(54,8 \%)$, pasando por sentirse libre (64\%) y ser más curioso (61\%).

Por su parte, dentro de los indicadores de estímulo, aunque el 51,6\% de los encuestados consideró que el coro contribuye muchísimo a intentar retos desafiantes, el resto de los ítems obtuvieron resultados más diversos. Particularmente en los que se hablaba acerca del papel del coro en hacer la vida más variada, excitante, arriesgada, generar nuevos retos y sensaciones. Aquí, los porcientos más altos estuvieron distribuidos de manera desigual. En el grado de muchísimo, ser arriesgado con un $41,9 \%$, en el grado de mucho, tener una vida excitante un $45,2 \%$, y para darle variedad a la vida, con un $35,5 \%$, también en mucho. 
Si se toma en cuenta que, autodirección y estímulo, son valores que pertenecen a la misma dimensión, se comprenderá que la compatibilidad entre las motivaciones que los generan es mayor. Esta relación muestra un interés especial por la novedad y la maestría.

A continuación, aparece universalismo, que queda visiblemente distanciado del resto de los valores hacia abajo (Fig.2). Contiene, por un lado, el ítem que mayor porciento de encuestados consideró que el coro influía muchísimo y por otro, una mayoría de indicadores que manifestaron valores mucho más bajos, que oscilan entre el $19,4 \%$ y el $25,8 \%$, yendo desde proteger el entorno $(19,4 \%)$, tener conocimiento del mundo y estar unido a la naturaleza $(22,6 \%)$, hasta ser socialmente justo $(25,8 \%)$.

En los últimos dos escaños aparecen poder y seguridad (Fig.2). Estos dos valores también son contiguos, por lo tanto, comparten la motivación de evitar o superar amenazas controlando las relaciones y los recursos. De los items que abordaban el poder, el que cuestiona acerca de conseguir riqueza fue señalado por el $61,2 \%$ entre nada y algo. Hay que considerar que la mayoría de los encuestados participan en el coro como parte de su currículo universitario o como actividad extracurricular, sin recibir remuneración alguna por realizarla. En otros indicadores de poder que hacen referencia a la autoridad, el reconocimiento social y la preservación de la imagen pública, la mayoría seleccionó entre bastante y mucho. Preservar la imagen pública, fue catalogada entre muchísimo y bastante por el 87\%, poseer autoridad por el 74,1\%, lograr poder social por el 70\% y obtener reconocimiento social por el $64,5 \%$.

Dentro de los indicadores de seguridad, valor que ocupa el punto más bajo, se les cuestionaba acerca de la contribución del coro en el ámbito del orden social, la seguridad de la familia y la nación, así como de la ética y la reciprocidad. En este caso, los resultados fueron más heterogéneos. Si bien, la gran mayoría consideró que el coro influía entre bastante y muchísimo a ser éticos $(93,5 \%)$, el resto de los items presentaron porcientos desiguales, distribuidos entre todas las opciones dadas.

Llegado este punto vale destacar que, de las 50 preguntas de la encuesta, 14 no fueron señaladas en los tres valores más bajos de la escala dada (nada, casi nada y poco). De manera más específica se observa que, 33 preguntas no fueron marcadas en nada y de las 17 en que esto ocurrió, 13 fueron por una sola persona.

Tomando como referencia algunos planteamientos de Schwartz (2012) acerca de los requisitos del funcionamiento social para analizar la jerarquía que se manifiesta en este grupo, es posible decir que la búsqueda del hedonismo (1) no necesariamente debe considerarse una amenaza a las relaciones sociales positivas dentro de un grupo, puesto que legitiman la necesidad innata de los seres humanos de obtener placer.

Conformidad (2), benevolencia (3) y tradición (4), contribuyen a las relaciones sociales positivas y cooperativas, requisito indispensable para el correcto funcionamiento social. Aunque el esfuerzo por lograr aprobación social puede interferir en el objetivo del grupo, los aspectos de logro (5) motivan a los individuos a implicarse en actividades grupales donde está contemplada su superación, mientras que los de autodirección (6) fomentan la creatividad y motivan la innovación. Con estímulo (7), también se legitima la necesidad de alcanzar placer.

Universalismo (8) es especialmente útil, cuando no existe una fácil identificación grupal. El poder (9), puede dañar las relaciones interpersonales, aunque la seguridad (10), también promueve relaciones sociales armoniosas.

El análisis de los valores porcentuales permitió detectar que el mismo número de encuestados consideró que el coro desarrollaba muchísimo, indicadores pertenecientes, tanto a valores de Conservación (conformidad y tradición), como a su opuesto Apertura al cambio (autodirección y estímulo). Por su parte valores de Autotrascendencia (benevolencia y universalismo) se ubican en lugares opuestos. Benevolencia, entre los 5 valores de mayor importancia, y universalismo, en los 5 de menor. Logro y poder, que pertenecen a Autopromoción, ocupan el quinto y noveno lugar, respectivamente.

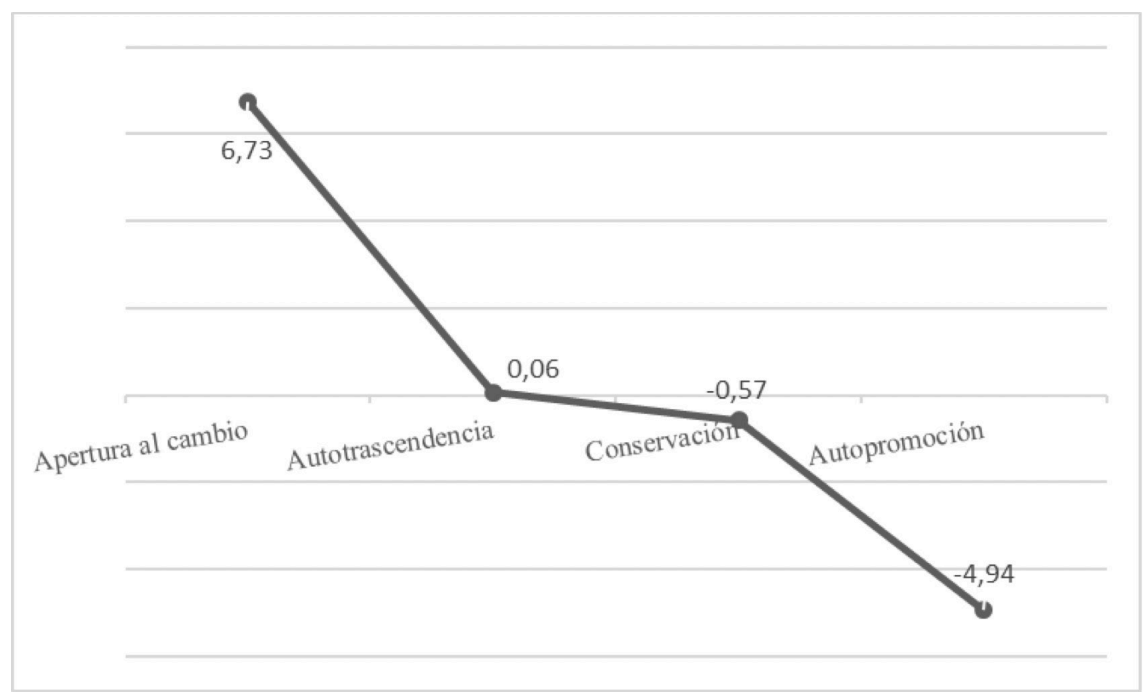

Figura 3. Jerarquía de dimensiones para grupo de cantores 
De esta forma, una vez procesada la información, la jerarquía que se establece para las dimensiones en este estudio, prioriza la Apertura al cambio como la dimensión que más se desarrolla dentro de la práctica coral. Seguidamente, a una distancia considerable, está Autotrascendencia. Posteriormente Conservación y, por último, Autopromoción (Fig.3).

\section{Discusión y conclusiones}

Acerca del valor formativo del coro se han realizado múltiples investigaciones en los últimos veinte años, en países como España, Estados Unidos, Reino Unido, Colombia, Venezuela, Israel y Noruega, centradas en agrupaciones de diversa índole, tanto profesionales como aficionadas, infantiles, universitarias, de adultos mayores, e incluso, con personas que poseen necesidades especiales y colectivos en riesgo de exclusión social (Narain, 2016). Muchas de ellas, a decir del autor, resaltan la influencia de la práctica coral en la educación y demuestran su papel en la formación de valores. Así mismo, Fernández (2014) confirma que varios estudios coinciden en que dentro del coro emergen valores perdurables que se reflejarán posteriormente en una sociedad saludable.

En concordancia con lo antes expuesto, esta investigación también demuestra que es posible desarrollar valores dentro de la práctica coral. En este caso específico, se toma como base la Teoría de los Valores de Schwartz y se establece una jerarquía basada en el análisis de las opiniones de los cantores de una agrupación universitaria en particular.

Como resultado del análisis se pudo determinar que hedonismo es el valor al que la práctica coral contribuyó en mayor medida. Seguidamente, aparecen tres puntos importantes dentro de esta jerarquía de manera descendente. Primero, la conjunción de conformidad, benevolencia y tradición. Esta expresa que los coristas reconocen que el coro les ha ayudado en gran medida a desarrollar el respeto por las normas que favorecen las relaciones cercanas y la inclinación hacia el grupo. En segundo lugar, en un punto medio, les ha permitido desarrollar sus capacidades e incentivado a la búsqueda de lo nuevo (autodirección y estímulo) y, en tercer lugar, la menor contribución estuvo en poder y seguridad, lo cual indica que les ha servido en menor grado, a evitar o superar peligros.

Otro aspecto del análisis relacionado con las dimensiones indicó que Apertura al cambio, que incluye hedonismo, autodirección y estimulo, se coloca como la dimensión que más han desarrollado dentro del coro, mientras que Autopromoción, que contempla poder y logro, aparece en el nivel más bajo.

Indagar acerca de la práctica coral, considerándola más allá del hecho estético que constituye, permite aproximarse a su relación con otras facetas del desempeño humano. "Los aspectos sociales encontrados científicamente en la práctica de la música coral la han dotado de una notable valía para la educación general y como no podía ser de otra forma, para la educación musical" (Pérez-Aldeguer 2014b, p.401). En consecuencia, se visibiliza la idoneidad de sus potencialidades para la formación de individuos integrales, plenos y cada vez más conscientes de la interdependencia en el mundo de hoy.

\section{Referencias bibliográficas}

Almenara, J. C. (2003). Educación en valores y cine. Making of. Cuadernos de cine y educación, 20, 16-30.

Aparicio, G. R. (2009). La educación en valores a través del teatro. Hekademos: revista educativa digital, (2), 85-98.

Cabedo, A. y Arriaga, C. (2016). ¿Música para aprender, música para integrar? Arte y educación en valores en el currículo escolar. Dedica. Revista de Educação e Humanidades, (9), 145-160.

Calderón, D., Oriola, S. y Gustems, J. (2015). Liderazgo y música: la figura del director. Artseduca, (10), 16-27.

Castro, B., Neto, M., Pena, L., \& Ribeiro, M. (2017). Valores: Una revisión de la literatura. Mediaciones Sociales, (16), $211-230$. https://doi.org/10.5209/meso.58117

Cayón, A. (2008). Estructura de Valores de Schwartz en el personal directivo universitario privado. Telos, 10(3), $403-417$.

Chica, C. A. B. (2008). Los ejes transversales como instrumento pedagógico para la formación de valores. Revista Iberoamericana de Educación, 45(2), 1-7. https://doi.org/10.35362/rie4522146

Conejo, P. A. (2012). El valor formativo de la música para la educación en valores. Dedica. Revista de Educação e Humanidades, (2), 263-278.

Cornago, Ó. (2010). Artes y Humanidades: una cuestión de formas (de hacer). Telón de Fondo, Revista de Teoría y Crítica Teatral, (12) $1-21$.

Expósito, C. D. (2018). Valores básicos del profesorado: una aproximación desde el modelo axiológico de Shalom Schwartz. Educación y educadores, 21(2), 3. http://dx.doi.org/10.5294/edu.2018.21.2.7

Fernández, N. S. (2014). Las agrupaciones corales y su contribución al bienestar de las personas: percepción de las aportaciones del canto coral a través de una muestra de cantores. Tesis doctoral. Universidad Carlos III de Madrid, España.

Ferrer, R. (2009). El canto coral y las orquestas infantiles, una educación en valores. Eufonía: Didáctica de la música, (45), 30-38.

Gainza, V. (2003). La educación musical entre dos siglos: del modelo metodológico a los nuevos paradigmas. Victoria-Buenos Aires: Universidad de San Andrés. Recuperado de http://hdl.handle.net/10908/773

Gallardo, J. (2011). Gestualidad y música coral. Trilogía Ciencia Tecnología Sociedad, 3(5), 141-148. http://dx.doi. org/10.22430/21457778.460 
García, V. A., Barbero, F. L. y Muñoz, R. C. (2017). Evaluación de la jerarquía de los valores humanos de Schwartz en la adolescencia: diferencias de género e implicaciones educativas. Revista Brasileira de Educação, 22(68), 123-146. http://dx. doi. org/10.1590/s1413-24782017226807.

Gervilla, E. (2004). Buscando valores: El análisis de contenido axiológico. Perfiles educativos, 26(103), 95-110.

Jaraba, M. (1989). Teoría y práctica del canto coral. Madrid, España: Editorial Alpuerto.

Joseph, D. y Southcott, J. (2017). Older people in a community gospel choir: musical engagement and social connection. Qualitative report, 22(12), 3209-3223.

Junco, J. G., Susanibar, E. M. y Dutschke, G. (2010). Una revisión exploratoria del modelo de Schwartz. Economía, Gestión y Desarrollo, 9, 35-66.

Lamont, A., Murray, M., Hale, R. y Wright-Bevans, K. (2018). Singing in later life: The anatomy of a community choir. Psychology of Music, 46(3), 424-439. https://doi.org/10.1177/0305735617715514

Lenger, D. (2013). El coro como herramienta de transformación social. I Congreso Coral Argentino, Mar del Plata, Argentina.

Lorenzo, M. (2017). Educación en valores en el contexto de las agrupaciones instrumentales y vocales 10 ideas prácticas para el aula. Artseduca, (17), 150-175.

Nadal, I., López, M. B., Fernández, C. y Juan, B. (2018). El coro Cantatutti: una práctica musical inclusiva en la Universidad de Zaragoza. En: Roig-Vila, Rosabel (ed.). El compromiso académico y social a través de la investigación e innovación educativas en la Enseñanza Superior (pp. 859-869). Barcelona, España: Octaedro.

Narain, J. (2016). La influencia de la música en la educación en valores: El valor formativo del coro. Universidad de Córdoba, España. Recuperado de https://www.researchgate.net/profile/Juan_Narain_Lucena/publication/311518257

Ojalvo, V., Kraftchenho, O., González, V. y Rojas, A. R. (2003). Conceptualización general de los valores. Pedagogía Universitaria, 8(1), 2-38.

Ordás, M. A., y Martínez, I. C. (2018). Voces colonizadas en América Latina. Decolonizando la ontología de la práctica coral. Arte e Investigación, (14), 9-20. https://doi.org/10.24215/24691488e009

O’Reilly, M. (2015). Análisis de la práctica coral desde la perspectiva de la formación de la competencia de trabajo en equipo. Revista Musica Hodie, 15(1), 25-30. https://doi.org/10.5216/mh.v15i1.39344

Páez,A.C.(2009). Literatura, pedagogía y formación en valores. Enunciación, 14(2), 5-20.https://doi.org/10.14483/22486798.3057

Parra, J. M. (2003). La educación en valores y su práctica en el aula. Tendencias pedagógicas, 8, 69-88.

Pérez-Aldeguer, S. (2014a). La música como herramienta para desarrollar la competencia intercultural en el aula. Perfiles educativos, 36(145), 175-187. https://doi.org/10.22201/iisue.24486167e.2014.145.45994

Pérez-Aldeguer, S. (2014b). El canto coral: una mirada interdisciplinar desde la educación musical. Estudios pedagógicos (Valdivia), 40(1), 389-404. http://dx.doi.org/10.4067/S0718-07052014000100023

Piotrowski, B. (2009). La axiología y la educación. Educación y educadores, 3, 127-138.

Schwartz, S. H. (1992). Universals in the content and structure of values: Theoretical advances and empirical tests in 20 countries. Advances in experimental social psychology, 25(1), 1-65. https://doi.org/10.1016/S0065-2601(08)60281-6

Schwartz, S. H. (2003). A proposal for measuring value orientations across nations. Questionnaire Package of the European Social Survey, 259(290), 259-319.

Schwartz, S. H. (2012). An overview of the Schwartz theory of basic values. Online readings in Psychology and Culture, 2(1), 2307-0919. https://doi.org/10.9707/2307-0919.1116

Tavares, P. S (2018). Prosódia e subtexto: uma pesquisa-ação sobre o texto na música vocal e coral e recomendações. Tesis de Maestría. Universidad de Brasilia, Brasil. 